\title{
The fate of subsolid nodule: predictable or unpredictable?
}

\author{
Yun-Ju Wu ${ }^{1}$, Jeng-Hwei Tseng ${ }^{2}$, Chia-Hao Liang ${ }^{3,4}$, En-Kuei Tang ${ }^{5,6}$, Fu-Zong Wu ${ }^{1,4,6}$ \\ ${ }^{1}$ Department of Radiology, Kaohsiung Veterans General Hospital, Kaohsiung; ${ }^{2}$ Department of Radiology, Chang Gung Memorial Hospital, Chang \\ Gung University, Taipei; ${ }^{3}$ Department of Biomedical Imaging and Radiological Sciences, National Yang-Ming University, Taipei; ${ }^{4}$ Faculty of \\ Medicine, School of Medicine, National Yang-Ming University, Taipei; ${ }^{5}$ Department of Surgery, Kaohsiung Veterans General Hospital, Kaohsiung; \\ ${ }^{6}$ Department of Nursing, Shu-Zen Junior College of Medicine and Management, Kaohsiung \\ Correspondence to: Fu-Zong Wu, MD. Division of thoracic and cardiovascular imaging; Department of Radiology at Kaohsiung Veterans General \\ Hospital, No. 386, Ta-Chung 1st Road, Kaohsiung City. Email: cmvwu1029@gmail.com. \\ Provenance and Peer Review: This is an invited article commissioned and reviewed by the Section Editor Jun Zhou (Department of Nuclear Medicine, \\ Zhongshan Hospital, Fudan University, Shanghai, China). \\ Response to: Hwang EJ, Park CM. Persistent pulmonary subsolid nodules: How long should they be observed until clinically relevant growth occurs? \\ J Thorac Dis 2019;11:S1408-11.
}

Submitted Dec 18, 2019. Accepted for publication Jan 08, 2020.

doi: $10.21037 /$ jtd.2020.01.56

View this article at: http://dx.doi.org/10.21037/jtd.2020.01.56

We thank Dr. Hwang et al. from Korea for their thoughtful comments on our recent article (1). Our group recently published a study in the Heart, Lung and Circulation, reporting that natural course of persistent subsolid nodules (SSNs) in the term of true growth, substantial growth and stage shift (2). In this retrospective single-center study, we enrolled 128 persistent SSNs [93 pure ground-glass nodules (GGNs) and 35 part-solid nodules (PSNs)] $3 \mathrm{~cm}$ or smaller from 128 participants, and reported their observation during long-term follow-ups (average of 3.57 years, maximum follow-up period of 12 years). In this study, pure GGNs exhibited median natural progression times of 7, 9, and 12 years for true growth, substantial growth, and stage shift, respectively. However, PSNs exhibited median natural progression times of 3, 3, and 9 years for true growth, substantial growth, and stage shift, respectively.

In recent years, increasing utilization of low-dose computed tomography (CT) tools for lung cancer screening has resulted in a significant portion of SSNs detected in the screening population, especially in an Asian population with the high prevalence of non-smoking related lung cancer $(3,4)$. Overall, most patients (approx. $70 \%$ ) with lung adenocarcinoma were non-smokers in Taiwan, and most patients with clinical symptoms are diagnosed at an advanced stage with poor survival outcome despite recent developments in the target therapy of late stage lung cancer over recent years (5). In the meantime, recent studies demonstrated that almost over $90 \%$ of screened lung cancers were stage I lung cancer (mostly adenocarcinoma spectrums) with the excellent prognosis in the Asian nonsmoking related lung cancer screening programs $(3,6)$.

The difference between non-screened and screened non-smoking related lung cancers make us notice the heterogeneity in growth pattern and risk factors of lung cancer (7).

According to recent literature reviews, there were high prevalence of non-smoking related lung cancers found in the Asian lung cancer screening program $(3,4,6)$. Most patients with lung cancers diagnosed in the screening program were not eligible for National Lung Screening Trial (NLST) criteria $(3,8)$. Previous studies have demonstrated that family history and female gender are two important risk factors with non-smoking related lung cancer $(3,4)$. In addition, the average PM2.5 levels in Taiwan were much higher than the levels in European cities according to the European Study of Cohorts for Air pollution Effects (ESCAPE), especially in Kaohsiung city (9). This issue of expanding screening eligibility criteria may be relevant in Taiwan because of regional genetic and environmental differences. Should never-smokers at increased risk for lung cancer be screened? Recent microsimulation study by Kelvin demonstrated that never-smokers with high risk (RRs of 15 to 35 ) have similar to better trade-offs between benefits and harms compared with population eligible for 


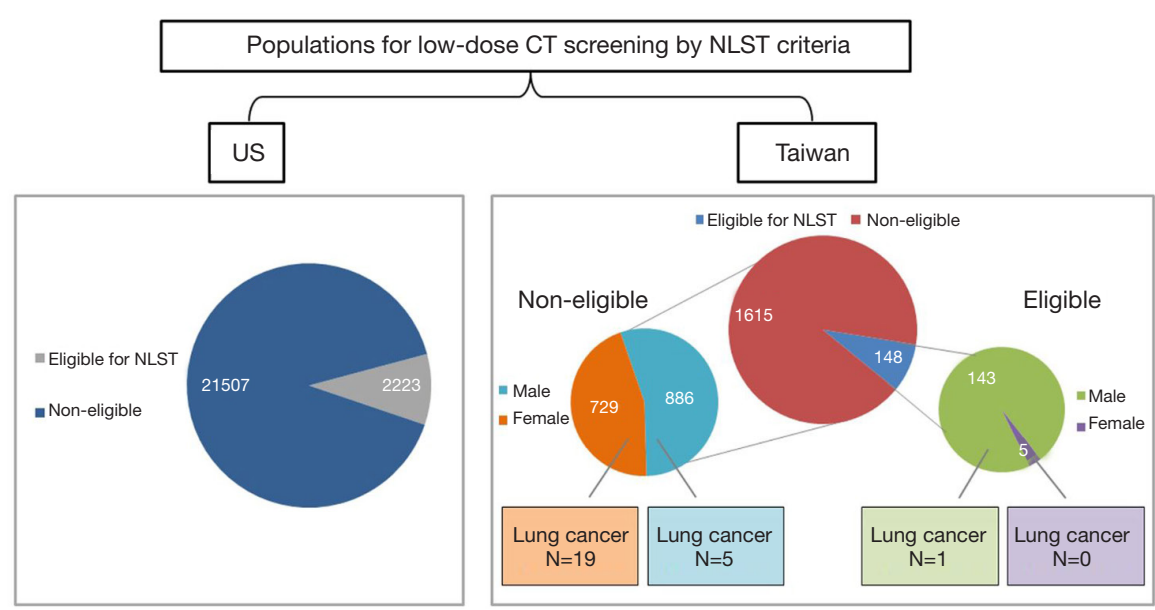

NLST criteria: Age 55-74 years with 30 or more pack-years of cigarette smoking or quit within 15 years packs per day years smoked or quit within 15 years.

Figure 1 Comparison of eligible populations for low-dose CT screening by NLST criteria in the US and Taiwan. CT, computed tomography; NLST, National Lung Screening Trial.

NSLT criteria (10). However, lung cancer screening is not beneficial for most never-smokers at low risk. Comparison of eligible populations for low-dose CT screening by NLST criteria in the US and Taiwan are summarized in Figure 1 according to previous several studies $(3,8,11,12)$. In the future, it is mandatory to investigate the proportion of real-world data on the rate of non-smoking related lung cancer in the United State and prognostic benefit in Asian non-smoking LDCT screening program.

There is a high prevalence of SSNs variant in size in the Asian non-smoking related population than in the US according to the previous studies. (16.2\% in Taiwan versus $3.7-4.2 \%$ in the US) $(3,13,14)$. However, this is the dilemma in clinical management. Over-management/overtreatment these small SSNs could lead to overdiagnosis (15). On the other hand, some patients are very anxious about these SSN lesions and worry that follow-up policy will lead to delays in lung cancer diagnosis and treatment. In this study, most GGNs have the more indolent behavior of growth pattern. Our study results are in line with 2017 Fleischer guideline recommendation (16). Wait and see policy with longer follow-up period $>5$ year is recommended for GGN management (2). For PSNs, they have the more aggressive behavior of growth pattern than GGNs. In addition, our study result showed that substantial growth is the high correlative with the degree of invasiveness, suggestive of substantial growth reflects the pathological invasive component of adenocarcinoma. Recent studies have demonstrated that older age, history of lung cancer, initial size $8 \mathrm{~mm}$ or larger, presence of a solid component, and air bronchogram were independent risk factors for subsequent SSN growth $(2,17)$. For these pure GGNs, the most important thing is to predict potential rapid-growing lesions. In the future, to develop the prediction model integrated with clinical characters, texture or volumetric analysis on radiomic features and genetic information for GGN growth prediction is warranted $(18,19)$.

\section{Acknowledgments}

Funding: None.

\section{Footnote}

Conflicts of Interest: The authors have no conflicts of interest to declare.

Ethical Statement: The authors are accountable for all aspects of the work in ensuring that questions related to the accuracy or integrity of any part of the work are appropriately investigated and resolved.

Open Access Statement: This is an Open Access article distributed in accordance with the Creative Commons Attribution-NonCommercial-NoDerivs 4.0 International License (CC BY-NC-ND 4.0), which permits the noncommercial replication and distribution of the article with 
the strict proviso that no changes or edits are made and the original work is properly cited (including links to both the formal publication through the relevant DOI and the license). See: https://creativecommons.org/licenses/by-nc-nd/4.0/.

\section{References}

1. Hwang EJ, Park CM. Persistent pulmonary subsolid nodules: How long should they be observed until clinically relevant growth occurs? J Thorac Dis 2019;11:S1408-11.

2. Tang EK, Chen CS, Wu CC, et al. Natural History of Persistent Pulmonary Subsolid Nodules: Long-Term Observation of Different Interval Growth. Heart Lung Circ 2019;28:1747-54.

3. Wu FZ, Huang YL, Wu CC, et al. Assessment of Selection Criteria for Low-Dose Lung Screening CT Among Asian Ethnic Groups in Taiwan: From Mass Screening to Specific Risk-Based Screening for Non-Smoker Lung Cancer. Clin Lung Cancer 2016;17:e45-56.

4. Lin KF, Wu HF, Huang WC, et al. Propensity score analysis of lung cancer risk in a population with high prevalence of non-smoking related lung cancer. BMC Pulm Med 2017;17:120.

5. Zhou F, Zhou C. Lung cancer in never smokers-the East Asian experience. Transl Lung Cancer Res 2018;7:450-63.

6. Hsu HT, Tang EK, Wu MT, et al. Modified LungRADS Improves Performance of Screening LDCT in a Population with High Prevalence of Non-smoking-related Lung Cancer. Acad Radiol 2018;25:1240-51.

7. Wu FZ, Kuo PL, Huang YL, et al. Differences in lung cancer characteristics and mortality rate between screened and non-screened cohorts. Sci Rep 2019;9:19386.

8. Aberle DR, Adams AM, Berg CD, et al. Reduced lungcancer mortality with low-dose computed tomographic screening. N Engl J Med 2011;365:395-409.

9. Tseng CH, Tsuang BJ, Chiang CJ, et al. The Relationship Between Air Pollution and Lung Cancer in Nonsmokers in Taiwan. J Thorac Oncol 2019;14:784-92.

10. Ten Haaf K, de Koning HJ. Should Never-Smokers at Increased Risk for Lung Cancer Be Screened? J Thorac Oncol 2015;10:1285-91.

11. Howard DH, Richards TB, Bach PB, et al. Comorbidities, smoking status, and life expectancy among individuals eligible for lung cancer screening. Cancer 2015;121:4341-7.

12. Yang D, Liu Y, Bai C, et al. Epidemiology of lung cancer and lung cancer screening programs in China and the United States. Cancer Lett 2020;468:82-7.

13. Yip R, Henschke CI, Xu DM, et al. Lung Cancers Manifesting as Part-Solid Nodules in the National Lung Screening Trial. AJR Am J Roentgenol 2017;208:1011-21.

14. Yankelevitz DF, Yip R, Smith JP, et al. CT Screening for Lung Cancer: Nonsolid Nodules in Baseline and Annual Repeat Rounds. Radiology 2015;277:555-64.

15. Wu FZ, Kuo PL, Wu CC, et al. The impact of patients' preferences on the decision of low-dose computed tomography lung cancer screening. Transl Lung Cancer Res 2018;7:S236-8.

16. MacMahon H, Naidich DP, Goo JM, et al. Guidelines for Management of Incidental Pulmonary Nodules Detected on CT Images: From the Fleischner Society 2017. Radiology 2017;284:228-43.

17. Kakinuma R, Noguchi M, Ashizawa K, et al. Natural History of Pulmonary Subsolid Nodules: A Prospective Multicenter Study. J Thorac Oncol 2016;11:1012-28.

18. Wu FZ, Chen PA, Wu CC, et al. Semiquantative Visual Assessment of Sub-solid Pulmonary Nodules $\leqq 3 \mathrm{~cm}$ in Differentiation of Lung Adenocarcinoma Spectrum. Sci Rep 2017;7:15790.

19. She Y, Zhang L, Zhu H, et al. The predictive value of CTbased radiomics in differentiating indolent from invasive lung adenocarcinoma in patients with pulmonary nodules. Eur Radiol 2018;28:5121-8.
Cite this article as: $\mathrm{Wu} \mathrm{YJ}$, Tseng $\mathrm{JH}$, Liang $\mathrm{CH}$, Tang EK, Wu FZ. The fate of subsolid nodule: predictable or unpredictable? J Thorac Dis 2020;12(3):1118-1120. doi: 10.21037/ jtd.2020.01.56 